Pacific Journal of Mathematics

THE BOUNDARY OF A SIMPLY CONNECTED DOMAIN AT AN 


\title{
THE BOUNDARY OF A SIMPLY CONNECTED DOMAIN AT AN INNER TANGENT POINT
}

\author{
JoHn MARAFINO
}

Let $T^{*}$ be the set of accessible boundary points at which the inner tangent to $\partial D$ exists. That is, if $a \in T^{*}$ and $w(a)$ represents its complex coordinate, then there exists a unique $\nu(a), 0 \leq \nu(a)<2 \pi$, such that for each $\varepsilon>0 \quad\left(\varepsilon<\frac{\pi}{2}\right)$ there exists a $\delta>0$ such that

$$
\begin{aligned}
\Delta=\left\{w(a)+\rho e^{l \nu}: 0<\rho<\delta,|\nu-\nu(a)|<\frac{\pi}{2}-\varepsilon\right\} \subset D \\
\text { and } w \rightarrow a \text { as } w \rightarrow w(a), \quad w \in \Delta .
\end{aligned}
$$

Let $\gamma(a, r)$ represent the unique component of $D \cap\{|w-w(a)|=r\}$ that intersects the inner normal $\left\{w(a)+\rho e^{i \nu(a)}: \rho>0\right\}, L(a, r)$ denote the length of $\gamma(a, r)$ and set $A(a, r)=\int_{0}^{r} L\left(a, r^{\prime}\right) d r^{\prime}$. Finally let $A D^{*}$ be those points of $T^{*}$ at which a non-zero angular derivative exists.

Our main result is a purely geometrical proof of a theorem that describes the boundary of $D$ near $a \in T^{*}$. As a consequence we have

(1) a geometric description of the boundary of $D$ near almost every $a \in A D^{*}$ that is a generalization of the geometric behavior of a smooth curve,

(2) an answer on $T^{*}$ and hence on $A D^{*}$ of the two open questions and conjectures made by McMillan in $[3$, p. 739] concerning the length and area ratios

$$
\frac{L(a, r)}{2 \pi r} \text { and } \quad \frac{A(a, r)}{\pi r^{2}} \quad \text { as } r \rightarrow 0 .
$$

\section{Introduction.}

1.1. Many of the definitions introduced in $\S \S 1.1$ to 1.3 can be found in McMillan's papers.

Let $D$ be a simply connected plane domain, not the whole plane, and define on $D$ the relative metric $d_{D}$, the relative distance between two points of $D$ being defined as the infimum of the Euclidean diameters of curves that lie in $D$ and join these two points. Let $\left(D^{*}, d_{D^{*}}\right)$ be the completion of the metric space $\left(D, d_{D}\right)$. Now $D^{*}=D \cup A^{*}$ where $D$ is an isometric copy of $D$ in $D^{*}$ and $A^{*}$ is the set of accessible boundary points of $D$. Any limits involving accessible boundary points are taken in $d_{D^{*}}$. 
Let $T^{*}$ be the set of accessible boundary points of $D$ at which the inner tangent to $\partial D$ exists. That is, if $a \in T^{*}$ and $w(a)$ represents its complex coordinate, then there exists a unique $\nu(a), 0 \leq \nu(a)<2 \pi$, such that for each $\varepsilon>0(\varepsilon<\pi / 2)$ there exists a $\delta>0$ such that

$$
\begin{aligned}
\Delta=\left\{w(a)+\rho e^{i \nu}: 0<\rho<\delta,|\nu-\nu(a)|<\pi / 2-\varepsilon\right\} \subset D & \\
& \text { and } w \rightarrow a \text { as } w \rightarrow w(a), \quad w \in \Delta .
\end{aligned}
$$

With $\arg (w-w(a))$ defined and continuous in $D$, we let $R^{*}$ be the set of accessible boundary points of $D$ such that

$$
\begin{aligned}
& \liminf _{\substack{w \rightarrow a \\
w \in D}} \arg (w-w(a))=-\infty \quad \text { and } \\
& \limsup _{\substack{w \rightarrow a \\
w \in D}} \arg (w-w(a))=+\infty .
\end{aligned}
$$

Points of $R^{*}$ are often called twist points. Using a one-to-one conformal mapping $f$ of the unit disk onto $D$, one can establish using a result of Koebe a one-to-one correspondence between $A^{*}$ and a dense subset of measure $2 \pi$ of the unit circle. We shall say that a set $B^{*} \subset A^{*}$ is a $D$-conformal null set provided that it corresponds to a set of measure zero on $\{|z|=1\}$ under this correspondence. This definition is independent of the map $f$. Let $z=g(w)$ be the inverse of $f$ that maps $D$ one-to-one and conformally onto the unit disk. Then for each $a \in A^{*}$, the limit

$$
\lim _{\substack{w \rightarrow a \\ w \in D}} g(w)=g(a)
$$

exists. We say that $g(w)$ has a finite non-zero angular derivative at $a \in A^{*}$ provided there exists a finite non-zero complex number $g^{\prime}(a)$ such that for each Stolz angle $\check{A}$ at $a$ contained in $D$,

$$
\lim _{\substack{w \rightarrow a \\ w \in \tilde{A}}} g^{\prime}(w)=g^{\prime}(a)
$$

Let $A D^{*}$ be those points in $A^{*}$ at which $g$ has a finite non-zero angular derivative. For each $a \in A D^{*}$, it follows that $g^{\prime}$ has a finite non-zero asymptotic value along some curve ending at $a$. Since $f^{\prime}$ is normal [2, p. 50] we have by [7, p. 267] that $f^{\prime}$ has a finite nonzero asymptotic value along some path ending at $g(a)$ which in turn 
implies that $f$ is conformal at $g(a)$. Consequently, $a \in T^{*}$ and $A D^{*} \subset T^{*}$. The first part of McMillan's twist point theorem [2, p. 44] nicely ties together all the concepts introduced in this section. It states that $A^{*}=T^{*} \cup R^{*} \cup N^{*}$, where $N^{*}$ is a $D$-conformal null set and that $T^{*} \backslash A D^{*}$ is a $D$ conformal null set.

1.2. Let $B$ be a subset of the plane and $\varepsilon$ be an arbitrary positive number. Define

$$
\lambda(\varepsilon)=\inf \sum_{k} d_{k},
$$

where the infimum is taken over all countable coverings of $B$ by disks $\Delta_{k}$ of diameter $d_{k}<\varepsilon$. Clearly, $0 \leq \lambda(\varepsilon) \leq+\infty$ and $\lambda(\varepsilon)$ increases as $\varepsilon$ decreases so that

$$
\Lambda^{*}(B)=\lim _{\varepsilon \rightarrow 0} \lambda(\varepsilon) \quad\left(0 \leq \Lambda^{*}(B) \leq+\infty\right)
$$

exists. $\Lambda^{*}$ is a metric outer measure whose $\sigma$-field of measurable sets include the Borel sets $\left[9\right.$, p. 64]. Moreover, $\Lambda^{*}$ is outer regular relative to the class of $G_{\delta}$ sets. The restriction of $\Lambda^{*}$ to this $\sigma$-field is denoted by $\Lambda$ and is called either the linear measure or the one dimensional Hausdorff measure. The second part of McMillan's twist point theorem [2, p. 44] states that a subset of $T^{*}$ is $D$-conformal null if and only if the set of complex coordinates of its points has linear measure zero. Since $T^{*} \backslash A D^{*}$ is a $D$ conformal null set we have by this result that

$$
\Lambda\left(T^{*} \backslash A D^{*}\right)=0 .
$$

In the remainder of this paper we will restrict our attention to $T^{*}$. A proposition $P(a)$ will be said to hold for almost every $a \in T^{*}$ if $\left\{w(a): a \in T^{*}\right.$ and $P(a)$ is false $\}$ has linear measure zero. By (1) any proposition holding for almost every $a \in T^{*}$ will also hold for almost every $a \in A D^{*}$. Consequently, Theorem 1 , Corollaries 1 and 2 , and Theorem 2 in $\S 1.3$ can be restated using $A D^{*}$ in place of $T^{*}$.

1.3. The main result of this paper is a geometric proof of a theorem describing the behavior of $\partial D$ in a neighborhood of almost every $a \in T^{*}$. For each $a \in T^{*}$ and $r>0$, let $\alpha(a, r)$ be the measure of the largest angle such that the sector

$$
\left\{w(a)+\rho e^{i \nu}: 0<\rho<r,|\nu-\nu(a)|<\alpha(a, r)\right\} \subset D .
$$


If no such angle exists, set $\alpha(a, r)=0$. Note that for each $a \in T^{*}$ there exists an $r_{a}$ such that $\alpha(a, r)>0$ for $r<r_{a}$. For each $a \in T^{*}$ and $r<r_{a}$, let $\gamma(a, r)$ be the unique component of $D \cap\{|w-w(a)|=$ $r\}$ that intersects the inner normal $\left\{w(a)+\rho e^{i \nu(a)}: \rho>0\right\}$. We denote the length of $\gamma(a, r)$ by $L(a, r)$ and set $A(a, r)=\int_{0}^{r} L\left(a, r^{\prime}\right) d r^{\prime}$. Measurability of the integrand is shown in [3, p. 730].

For each $r<r_{a}$, we parameterize $\gamma(a, r)$ by $w_{r}(t)=w(a)+r e^{i t}$, $t_{0}(r)<t<t_{1}(r)$. For $S \subset\left(0, r_{a}\right)$, let $E_{L}^{*}(S)=\left\{\zeta \in A^{*}: w(\zeta)=\right.$ $w(a)+r e^{i t_{0}(r)}, r \in S$, and $w(a)+r e^{i t} \rightarrow \zeta$ as $\left.t \rightarrow t_{0}(r)^{+}\right\}$. That is, $E_{L}^{*}(S)$ is the set of accessible boundary points determined by $w_{r}(t)$ as $t \rightarrow t_{0}(r)^{+}, r \in S$. Similarly, $E_{R}^{*}(S)$ is the set of accessible boundary points determined by $w_{r}(t)$ as $t \rightarrow t_{1}(r)^{-}, r \in S$. Finally, set $E^{*}(S)=E_{L}^{*}(S) \cup E_{R}^{*}(S)$. In what follows $h(t)$ is a positive real valued function defined on $(0,+\infty)$ with the property that $\lim _{t \rightarrow 0} h(t)=0$. An interesting case is when $h(t)=k t$ for large positive $k$.

TheOREM 1. For almost every $a_{0} \in T^{*}$, there exists a set $S \subset$ $\left(0, r_{a_{0}}\right)$, closed relative to $\left(0, r_{a_{0}}\right)$, such that

(i) $E^{*}(S) \subset T^{*}$ and

$$
\lim _{r \rightarrow 0} \frac{m(S \cap(0, r))}{r}=1,
$$

where $m$ denotes Lebesgue measure.

(ii)

$$
\lim _{\substack{a \rightarrow a_{0} \\ a \in E_{R}^{*}(S)}} \arg \left(w(a)-w\left(a_{0}\right)\right)=\nu\left(a_{0}\right)+\pi / 2
$$

and

$$
\lim _{\substack{a \rightarrow a_{0} \\ a \in E_{L}^{*}(S)}} \arg \left(w(a)-w\left(a_{0}\right)\right)=\nu\left(a_{0}\right)-\pi / 2
$$

(iii)

$$
\lim _{\substack{a \rightarrow a_{0} \\ a \in E^{*}(S)}} \nu(a)=\nu\left(a_{0}\right) \quad \text { if } \nu\left(a_{0}\right) \neq 0
$$

and

$$
\lim _{\substack{a \rightarrow a_{0} \\ a \in E^{*}(S)}} \nu(a)=0 \quad(\bmod 2 \pi) \quad \text { if } \nu\left(a_{0}\right)=0 .
$$

$$
\lim _{\substack{a \rightarrow a_{0} \\ a \in E^{*}(S)}} \alpha\left(a, h\left(\left|w(a)-w\left(a_{0}\right)\right|\right)\right)=\pi / 2 .
$$


If the boundary of $D$ is a smooth Jordan curve, all boundary points of $D$ are accessible since, by a theorem of Carathéodory, any conformal mapping from $\{|z|<1\}$ to $D$ extends to a homeomorphism of $\{|z| \leq 1\}$ to $\bar{D}$. We need make no distinction between accessible boundary points of $D$ and the boundary of $D$. Recall that a curve is said to be smooth if its parameterization $z(t)$ has a continuous non-zero derivative everywhere. Thus at each point of $\partial D$ there is an inner tangent. So $T^{*}$ coincides with the boundary of $D$. Since the boundary of $D$ is a smooth curve, this implies that

(a) the curve has everywhere a continuously turning tangent (and normal)

(b) the oscillation of $z(t)$ near $z(a)$ must diminish as $t \rightarrow a$.

It is these two properties that (iii) and (iv) of Theorem 1 are characterizing. Clearly, at each point $a \in \partial D$ with $S=\left(0, r_{a}\right)$, properties (i)-(iv) of Theorem 1 hold. (A consideration of the smooth boundary of the unit disk illustrates the need for $h(t)$ in (iv) to tend to zero as $t$ tends to zero.) Hence, Theorem 1 offers a geometric description of the boundary of $D$ near almost every $a \in T^{*}$ that closely resembles the geometric behavior of a smooth curve. In $\S 1.4$ we will display an example that demonstrates how the smoothness property in (iv) restricts certain boundary behavior.

From the proof of this theorem the following results will be immediate:

COROllary 1. For almost every $a \in T^{*}$,

$$
\liminf _{r \rightarrow 0} \frac{L(a, r)}{2 \pi r}=\frac{1}{2}
$$

COROLlaRY 2. For almost every $a \in T^{*}$ and for every $\varepsilon>0$,

$$
\left\{r \in\left(0, r_{a}\right): \frac{L(a, r)}{2 \pi r} \geq \frac{1}{2}+\varepsilon\right\}
$$

has density zero at 0 ; that is, 0 is a point of dispersion of this set $[\mathbf{5}, p$. 184].

Using Corollary 2 we will then be able to show a secondary result concerning the area of $D$ near $a \in T^{*}$. 
TheORem 2. For almost every $a \in T^{*}$,

$$
\lim _{r \rightarrow 0} \frac{A(a, r)}{\pi r^{2}}=\frac{1}{2}
$$

1.4. We make a few comments and display some examples that highlight the properties of Theorems 1 and 2 and that lead to certain conclusions.

We first compare (i) and (ii) of Theorem 1 to Ostrowski's condition: We say that Ostrowski's condition holds at $a \in T^{*}$ if there exist sequences $\left\{a_{n}^{\prime}\right\}$ and $\left\{a_{n}^{\prime \prime}\right\}$ of accessible boundary points of $D$ tending to $a \in T^{*}$ for which the corresponding sequences of complex coordinates $\left\{w\left(a_{n}^{\prime}\right)\right\},\left\{w\left(a_{n}^{\prime \prime}\right)\right\}$ tending to $w(a)$ satisfy

$\left(\mathrm{i}^{\prime}\right) \arg \left(w\left(a_{n}^{\prime}\right)-w(a)\right) \rightarrow \nu(a)+\pi / 2, \arg \left(w\left(a_{n}^{\prime \prime}\right)-w(a)\right) \rightarrow \nu(a)-$ $\pi / 2$,

$\left(\mathrm{ii}^{\prime}\right)$

$$
\frac{\left|w\left(a_{n+1}^{\prime}\right)-w(a)\right|}{\left|w\left(a_{n}^{\prime}\right)-w(a)\right|} \rightarrow 1, \quad \frac{\left|w\left(a_{n+1}^{\prime \prime}\right)-w(a)\right|}{\left|w\left(a_{n}^{\prime \prime}\right)-w(a)\right|} \rightarrow 1 .
$$

Let $g(w)$ be a one-to-one conformal map of $D$ onto the open unit disk. Ostrowski's condition is necessary and sufficient for $g(w)$ to be isogonal (or conformal) at a given point [6].

McMillan observes in [4, pp. 68, 73] that at each $a \in A D^{*}, g(w)$ is isogonal and as a consequence Ostrowski's condition holds. This observation is reflected in Theorem 1, parts (i) and (ii). It states that at almost every $a \in A D^{*}$ a condition slightly stronger than Ostrowski's holds. In the following two examples we illustrate how this condition restricts certain boundary behavior.

EXAMPLE 1. Let $B^{*} \subset T^{*}$ be such that the local behavior of $\partial D$ near $a_{0} \in B^{*}$ is similar to that shown in Figure 1 . Let $B=$ $\left\{w\left(a_{0}\right): a_{0} \in B^{*}\right\}$ and for each $a_{0} \in B^{*}$ define $E_{R}(S)=\{w(a): a \in$ $\left.E_{R}^{*}(S)\right\}$. We want to show $\Lambda(B)=0$. Note that Ostrowski's condition holds on $B^{*}$. In fact any sequence $\left\{a_{n}^{\prime}\right\}$ where $a_{n}^{\prime}$ is on the boundary over the intervals $(1 / 2 k+1,1 / 2 k), k=1,2, \ldots$, satisfies the condition. Thus the mapping is isogonal at these points. In addition, using the results of Rodin and Warschawski [8, p. 5] there is a finite non-zero angular derivative at such points. If we use property (ii) of Theorem 1 , then $E_{R}(S)$ is on the boundary near or over the intervals $(1 / 2 k+1,1 / 2 k), k=1,2, \ldots$, and it follows that $S$ is contained in the intervals $(1 / 2 k+1,1 / 2 k), k=1,2, \ldots$, on the 


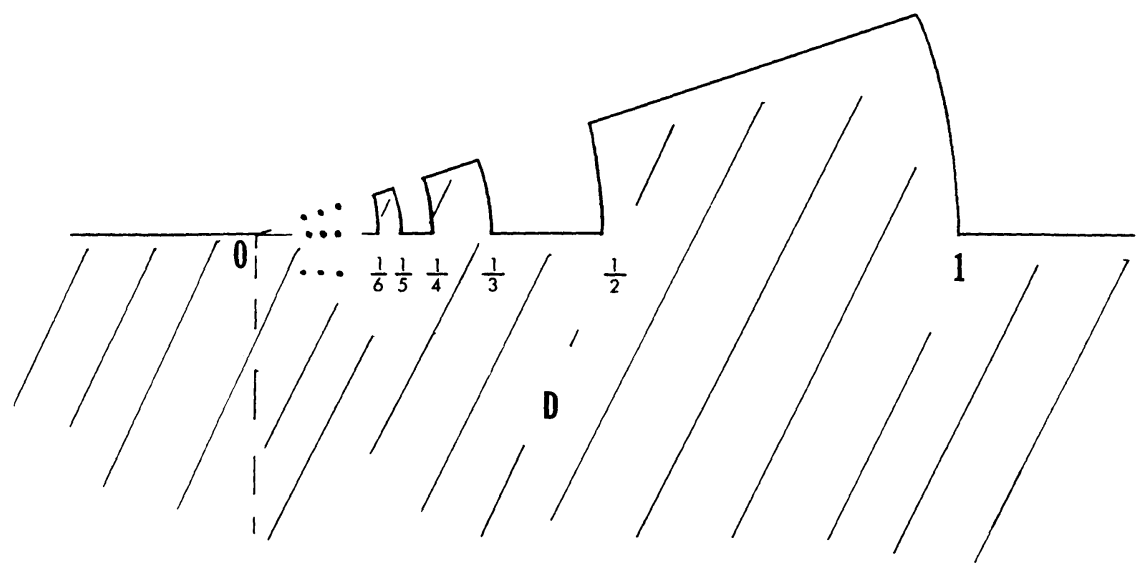

FigURE 1

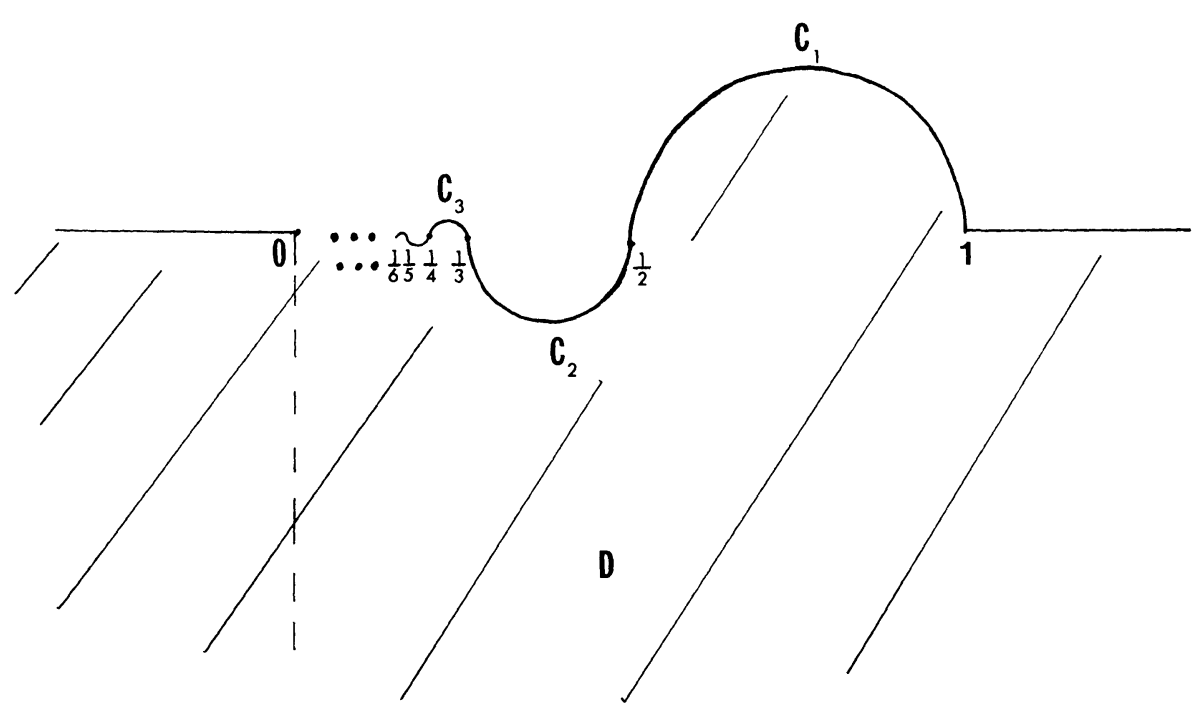

Figure 2

inner normal. Such a restriction violates (i) of Theorem 1 and hence Theorem 1 fails on $B^{*}$. Thus $\Lambda(B)=0$.

EXAMPLE 2. Let $B^{*} \subset T^{*}$ be such that the local behavior of $\partial D$ near $a_{0} \in B^{*}$ is similar to that shown in Figure 2 . The $C_{n}$ closely approximate circles. Let $B$ and $E_{R}(S)$ be as defined earlier. Again Ostrowski's condition holds and so the mapping is isogonal at these 
points. Once again the results of Warchawski and Tsuji [9, p. 366] show that a finite non-zero angular derivative exists at these points. Using property (iv) of Theorem 1 with $h(t)=100 t$ we see that $E_{R}(S)$ must be contained in the $C_{2 k}, k=1,2, \ldots$ Again the density property for $S$ is violated. Hence $\Lambda(B)=0$ by Theorem 1 .

EXAMPLE 3. So far we have demonstrated in our examples how Theorem 1 restricts certained boundary behavior where a non-zero angular derivative exists. We now construct a nontrivial example of boundary behavior that Theorem 1 does not restrict to sets of linear measure zero. We shall be working on the segment from $(0,0)$ to $(1,0)$ of the $x$ axis which we denote by $[0,1]$. Our domain $D$ will be the half plane $\operatorname{Im} z<0$ and anything we join to it from the construction. On the first step of the construction we remove the middle third of the segment $[0,1]$ and join there a rectangle $R_{1}$ of height $l_{1}=1 / 3$. We set $D_{1}=$ interior $\left(\{\operatorname{Im} z<0\} \cup R_{1}\right)$. At the beginning of the $m$ th stage of the construction we are left with $2^{m-1}$ segments of $[0,1]$. From the middle of each of these we remove a segment of length $d_{m}=2^{-m+1} 3^{-m}$ and join there a rectangle of height $l_{m}=\left(1+3^{m}\right) 3^{-m} 2^{-m-1}$. Let $R_{m, k}, k=1,2, \ldots, 2^{m-1}$, denote these rectangles and set $D_{m}=$ interior $\left(D_{m-1} \cup\left(\cup R_{m, k}\right)\right)$. We let $D=\bigcup D_{m}$ and we make no distinction between the accessible boundary points of $D$ and $\partial D$. Figure 3 displays stages of the construction near 0 . Note that $\partial D \cap[0,1]$ is a perfect set $P$ such that $P \subset T^{*}$ and the Lebesgue measure of $P$ is $1 / 2$. Let $B \subset P$ be such that each $p \in B$ is a point of density of $P$. By the Density Theorem [5, p. 187] the Lebesgue measure of $B$ is $1 / 2$ and it follows that $\Lambda(B)=1 / 2$. Using the results of McMillan's twist point theorem, we know a finite non-zero angular derivative exists at each point of $B$, with the possible exception of a set of linear measure zero. By removing such a set and relabeling $B$ we can assume that the angular derivative exists at each point of $B$. At each point $p \in B$ the intersection of the circular projections of $B \cap[p, 1]$ and $B \cap[0, p]$ onto the inner normal at $p$ is a set $S_{p}$ at which Theorem 1 holds. We have constructed an example of boundary behavior that Theorem 1 does not restrict to sets of linear measure zero. In this example we also have at almost every point $a \in T^{*}$,

$$
\lim _{r \rightarrow 0} \frac{A(a, r)}{\pi r^{2}}=\frac{1}{2}
$$




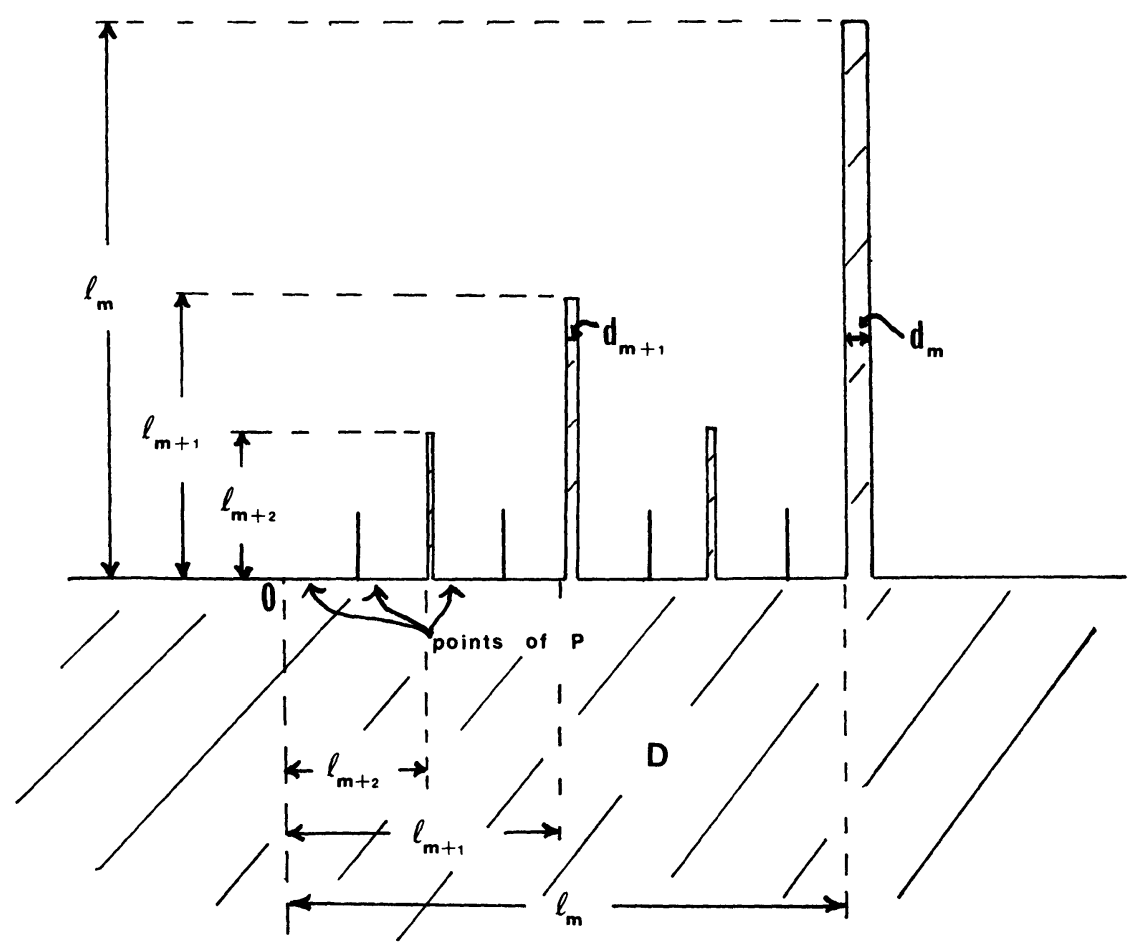

FIGURE 3

We finally consider Corollary 1 and Theorem 2. In [3] McMillan proved that except for a $D$-conformal null set of $A^{*}$,

$$
\limsup _{r \rightarrow 0} \frac{A(a, r)}{\pi r^{2}} \geq \frac{1}{2} \quad \text { and } \quad \limsup _{r \rightarrow 0} \frac{L(a, r)}{2 \pi r} \geq \frac{1}{2} .
$$

After considering a particular example, he then conjectured [3, p. 739], $\left[4\right.$, p. 74] that except for a $D$-conformal null set of $A^{*}$,

$$
\liminf _{r \rightarrow 0}, \frac{A(a, r)}{\pi r^{2}} \leq \frac{1}{2} \quad \text { and } \quad \liminf _{r \rightarrow 0} \frac{L(a, r)}{2 \pi r} \leq \frac{1}{2} .
$$

In this paper we not only confirm this conjecture on $T^{*}$ but also evaluate these quantities.

\section{Proof of Theorem 1 and of Theorem 2.}

\subsection{Let}

$$
F^{*}=\left\{a_{0} \in T^{*}: \text { for any relatively closed set } S \subset\left(0, r_{a_{0}}\right)\right.
$$

one of the properties (i)-(iv) fails

and $F=\left\{w\left(a_{0}\right): a_{0} \in F^{*}\right\}$. For any $B^{*} \subset F^{*}$, let $B=\{w(a): a \in$ $\left.B^{*}\right\}$. We must show that $\Lambda(F)=0$. 
2.2. Suppose to the contrary that $\Lambda^{*}(F)>0$. Since there exists a bounded subset $B$ of $F$ such that $\Lambda^{*}(B)>0$, we replace $F$ by such a subset and assume without loss of generality that $F$ is bounded.

Let $\varepsilon_{0}$ satisfy $0<\varepsilon_{0}<\pi / 10$. Associate with each $a \in F^{*}$ rational numbers $\alpha(a), \beta(a), \gamma(a)$ such that

$$
\begin{aligned}
& \pi / 2-\varepsilon_{0}<\alpha(a)<\pi / 2, \quad|\nu(a)-\beta(a)|<\varepsilon_{0} \quad \text { and } \\
& \Delta(a)=\left\{w(a)+\rho e^{i \nu}: 0<\rho<\gamma(a),|\nu-\beta(a)|<\alpha(a)\right\} \subset D .
\end{aligned}
$$

It follows that there exists a $B^{*} \subset F^{*}$ such that for all $a \in B^{*}$, $\alpha(a)=\alpha_{0}, \beta(a)=\beta_{0}, \gamma(a)=\gamma_{0}$, and $\Lambda^{*}(B)>0$. By replacing $F^{*}$ by $B^{*}$ we can assume without loss of generality that for all $a \in F^{*}$, $\alpha(a)=\alpha_{0}, \beta(a)=\beta_{0}$, and $\gamma(a)=\gamma_{0}$. Again associate with each $a \in F^{*}$ a straight $\ell(a)$ such that

$\ell(a)$ intersects the segment $\left\{w(a)+\rho e^{i \beta_{0}}: 0<\rho<\right.$ $\left.\gamma_{0}\right\}$ at right angles and the Euclidean distance from the origin to $\ell(a)$ is a rational number and one component of $\Delta(a) \backslash \ell(a)$ is triangular.

Since $\{\ell(a)\}$ is a countable set, there exists a $B^{*} \subset F^{*}$ such that for all $a \in B^{*}, \ell(a)=\ell_{0}$ and $\Lambda^{*}(B)>0$. We thus replace $F^{*}$ by $B^{*}$ and assume without loss of generality that for all $a \in F^{*}, \ell(a)=\ell_{0}$.

For each $a \in F^{*}$ we define $\Delta^{\prime}(a)$ to be the triangular component of $\Delta(a) \backslash \ell_{0}$. The set $\bigcup_{a \in F^{*}} \Delta^{\prime}(a)$ has at most countably many components, one of which has the form $G=\bigcup_{a \in B^{*}} \Delta^{\prime}(a)$ where $\Lambda^{*}(B)>0$. Replacing $F^{*}$ by $B^{*}$ we again assume without loss of generality that $G=\bigcup_{a \in F^{*}} \Delta^{\prime}(a)$ is connected. Thus, part of the boundary of $G$ is a closed segment lying on $\ell_{0}$ and the rest of the boundary is contained in one of the half planes determined by $\mathbf{C} \backslash \ell_{0}$. Without loss of generality we may assume that $\ell_{0}$ is the $x$ axis, $\ell_{0} \cap \partial G$, is the segment from $(0,0)$ to $(m, 0)$, which we denote by $[0, m]$, and $\partial G \backslash[0, m]$ lies in the upper half plane. Using the construction of $G$ and the fact that, $\frac{\pi}{2}-\alpha_{0}<\varepsilon_{0}<\frac{\pi}{10}$, one is able to define a function $f(x)$ on $[0, m]$ such that

$$
\begin{aligned}
& \partial G=\Gamma \cup[0, m] \quad \text { where } \Gamma=\{(x, f(x)): x \in[0, m]\} \quad \text { and } \\
& \left|f\left(x^{\prime}\right)-f\left(x^{\prime \prime}\right)\right| \leq \frac{1}{3}\left|x^{\prime}-x^{\prime \prime}\right| \quad \text { for all } x^{\prime}, x^{\prime \prime} \in[0, m] .
\end{aligned}
$$

It follows that $\partial G$ is a rectifiable Jordan curve that contains the point set $F \subset \Gamma$ and that $\Lambda^{*}(F)>0$. Let $\varepsilon=\Lambda^{*}(F)$.

We shall make no distinction between $\partial G$ and the set of accessible boundary points of $G$. 
We now construct a subset $B^{*}$ of $T^{*}$ so that

(i) $B$ is a closed, $\Lambda$-measurable subset of $\Gamma$ for which $\Lambda^{*}(B \cap F)$ $>0$

(ii) $\rho_{0}=\operatorname{dist}(B,[0, m])>0$

(iii) If $P$ is the projection map from $\Gamma \rightarrow[0, m]$ then $\left.f^{\prime}\right|_{P(B)}$ is continuous.

We have the inequalities $\Lambda^{*}(P(X)) \leq \Lambda^{*}(X) \leq 3 \Lambda^{*}(P(X))$ for any subset of $\Gamma$. The second inequality is true since $f$ is Lipschitz with character $1 / 3$. Since $f$ is Lipschitz, the subset of $[0, m]$ where $f$ is not differentiable has $\Lambda^{*}$ (and thus $\Lambda$ ) measure zero. Thus, by Lusin's theorem, $f^{\prime}$ is continuous on some closed subset $\widehat{B}$ of $[0, m]$ where $\Lambda^{*}([0, m] \backslash \widehat{B})<\varepsilon / 6$, and we may assume that $\widehat{B} \subset(0, m)$. Let $B$ be such that $P(B)=\widehat{B}$. Then

$$
\begin{aligned}
\Lambda^{*}(B \cap F) & \geq \Lambda^{*}(P(B \cap F)) \\
& =\Lambda^{*}(P(B) \cap P(F))=\Lambda^{*}(\widehat{B} \cap P(F)) \\
& \geq \Lambda^{*}(P(F))-\Lambda^{*}(P(F) \backslash \widehat{B}) .
\end{aligned}
$$

Now $\Lambda^{*}(P(F)) \geq \varepsilon / 3$ and $\Lambda^{*}(P(F) \backslash \widehat{B}) \leq \Lambda^{*}([0, m] \backslash \widehat{B}) \leq \varepsilon / 6$. Thus,

$$
\Lambda^{*}(B \cap F) \geq \frac{\varepsilon}{3}-\frac{\varepsilon}{6}=\frac{\varepsilon}{6} .
$$

A little thought shows that if $w \in \partial D \cap \partial G$ then there is a unique accessible boundary point $a$ of $D$, with complex coordinate $w$, which is accessible through $G$. Using this observation for each $w \in B$ defines a set $B^{*}$ with all the desired properties.

2.3. We have the following information on $B^{*}$ and $B$ :

(i) For each $a \in B^{*}$, define $N(w(a))=\operatorname{Arctan}\left(f^{\prime}(P(w(a)))\right)-\frac{\pi}{2}$. Since $\left.f^{\prime}\right|_{P(B)}$ is continuous, $N$ is continuous on $B$. Also, $N(w(a))$ satisfies the same inner tangent condition as $\nu(a)$. It follows from the uniqueness of $\nu(a)$ (recall the definition of $T^{*}$ ) that $N(w(a))=$ $\nu(a)$ and as a consequence $\nu(a)$ is continuous on $B^{*}$. Thus Property (iii) of Theorem 1 holds at each point $a$ of $B^{*}$. We now show that Property (ii) of Theorem 1 holds at each point $a$ of $B^{*}$. In fact, let $a_{0} \in B^{*}$.

Since $f^{\prime}$ is continuous at $P\left(w\left(a_{0}\right)\right)$,

$$
\lim _{\substack{P(w(a))>P\left(w\left(a_{0}\right)\right) \\ a \rightarrow a_{0}, a \in B^{*}}} \arg \left(w(a)-w\left(a_{0}\right)\right)=\arctan f^{\prime}\left(P\left(w\left(a_{0}\right)\right)\right)=\nu\left(a_{0}\right)+\frac{\pi}{2} .
$$


Similarly,

$$
\begin{aligned}
\lim _{\substack{P(w(a))<P\left(w\left(a_{0}\right)\right) \\
a \rightarrow a_{0}, a \in B^{*}}} & \arg \left(w(a)-w\left(a_{0}\right)\right) \\
= & \arctan f^{\prime}\left(P\left(w\left(a_{0}\right)\right)\right)-\pi=\nu\left(a_{0}\right)-\frac{\pi}{2} .
\end{aligned}
$$

(ii) For each $w \in B, w=w(a)$ with $a \in B^{*}$, and for each $r>0$, define $\alpha_{G}(w, r)$ to be the measure of the largest angle such that

$$
\left\{w+\rho e^{i \nu}: 0<\rho<r,|\nu-\nu(a)|<\alpha_{G}(w, r)\right\} \subset G \text {. }
$$

If no such angle exists, set $\alpha_{G}(w, r)=0$. Note that $\alpha_{G}(w, r)$ : is analogous to $\alpha(a, r)$ defined in $\S 1.3$. From the construction of $G$, $\alpha_{G}(w, r)$ is positive for all $w \in B$ and $r<\rho_{0}=\operatorname{dist}(B,[0, m])$. Fix $r<\rho_{0}$. After drawing a picture and using the continuity of $\nu(a)$ at $a_{0}$, one sees that $\alpha_{G}(w, r)$ is uppersemicontinuous at $w_{0}=w\left(a_{0}\right)$; that is,

$$
\lim _{\substack{w \rightarrow w_{0} \\ w \rightarrow w_{0} \in B}} \alpha_{G}(w, r) \leq \alpha_{G}\left(w_{0}, r\right) .
$$

We shall show that there exists a closed subset $\widetilde{B} \subset B$ such that $\Lambda^{*}(\widetilde{\boldsymbol{B}} \cap F)>0$ and for $w, w_{0} \in \widetilde{\boldsymbol{B}}$,

$$
\lim _{w \rightarrow w_{0}} \alpha_{G}\left(w, h\left(\left|w-w_{0}\right|\right)\right)=\pi / 2 .
$$

From the uppersemicontinuity of $\alpha_{G}$, for each $r<\rho_{0}, \alpha_{G}(w, r)$ is measurable on $B$. We let $\left\{r_{n}\right\}$ be a sequence of numbers such that $r_{n} \rightarrow 0$ and $r_{n}<\rho_{0}$ for all $n$. We define $\alpha_{n}(w)=\alpha_{G}\left(w, r_{n}\right)$ on $B$ for each $n$. The sequence of measurable functions $\left\{\alpha_{n}(w)\right\}$ defined on $B$ converges pointwise to the function $\alpha_{0}(w)=\pi / 2$. By Egoroff's Theorem [5, p. 108] there exists a measurable subset $B_{1} \subset B$ such that $\Lambda^{*}\left(B_{1} \cap F\right)>0$ and such that $\alpha_{n}(w)$ converges uniformly to $\alpha_{0}(w)=$ $\pi / 2$ on $B_{1}$. Since $B_{1}$ is measurable there exists a closed set $\widetilde{B} \subset B_{1}$, such that $\Lambda\left(B_{1} \backslash \widetilde{B}\right)$ is sufficiently small to ensure $\Lambda^{*}(\widetilde{B} \cap F)>0$. We now let $w, w_{0} \in \widetilde{B}$ and let $\varepsilon$ be an arbitrary positive number. Since $\left\{\alpha_{n}(w)\right\}$ converges uniformly on $\widetilde{B}$ to $\pi / 2$, there exists $N>0$ such that $\left|\pi / 2-\alpha_{N}(w)\right|<\varepsilon$ for all $w \in \widetilde{B}$. Let $w$ be sufficiently near $w_{0}$ to ensure that $h\left(\left|w-w_{0}\right|\right)<r_{N}$. Thus, for all $w \in \widetilde{B}$ sufficiently near $w_{0} \in \widetilde{B},\left|\pi / 2-\alpha_{G}\left(w, h\left(\left|w-w_{0}\right|\right)\right)\right| \leq\left|\pi / 2-\alpha_{G}\left(w, r_{N}\right)\right|<\varepsilon$.

(iii) By replacing $B$ with $\widetilde{B}$ one can assume without loss of generality that the properties listed in (i)-(ii) above hold on $B^{*}$ and $B$ and that $B$ is closed. In addition, since

$$
\alpha_{G}\left(w, h\left(\left|w-w_{0}\right|\right)\right) \leq \alpha\left(a, h\left(\left|w(a)-w\left(a_{0}\right)\right|\right)\right) \leq \pi / 2
$$


we have that

$$
\lim _{\substack{a \rightarrow a_{0} \\ a, a_{0} \in B^{*}}} \alpha\left(a, h\left(\left|w(a)-w\left(a_{0}\right)\right|\right)\right)=\pi / 2
$$

and this is property (iv) of Theorem 1 .

2.4. Since almost every point of $B \cap F$ is a point of density for $B \cap F$ and $\Lambda^{*}(B \cap F)>0$, there exists a point $w_{0}=w\left(a_{0}\right) \in B \cap F$ that is a point of density of the set. We fix this $w_{0}$ and consider the circular projection of $\Gamma$ onto the inner normal at $w_{0}$. (Recall that the tangent to $\Gamma$ exists at $w_{0}$.) Using the Lipschitz condition from $\S 2.2$ it can be shown $[1$, pp. 33,39$]$ that the restriction of the circular projection to that part of $\Gamma$ to the right of $w_{0}$, that is, the part of $\Gamma$ from $w\left(a_{0}\right)$ to $(m, f(m))$, is a one-to-one map and that $w_{0}$ is a point of density of the image of $B$ under such a map. Similarly, the restriction of the circular projection to that part of $\Gamma$ to the left of $w_{0}$ is one-to-one and $w_{0}$ is a point of density of the image of $B$ under such a map. As was done in Example 3 we define $S_{w_{0}}$ to be those points on the inner normal at $w_{0}$, whose distance from $w_{0}$ is less than $\rho_{0}$, that are the intersection of the circular projections of that part of $B \subset \Gamma$ to the right of $w_{0}$ and that part of $B \subset \Gamma$ to the left of $w_{0}$. Let $S=\left\{\rho: w_{0}+\rho e^{i \nu\left(a_{0}\right)} \in S_{w_{0}}\right\}$. It follows that $S$ is closed relative to $\left(0, \rho_{0}\right)$ and 0 is a point of density of $S$ on $\left(0, \rho_{0}\right)$. Letting $E_{R}(S)=\left\{w(a): a \in E_{R}^{*}(S)\right\}, E_{L}(S)=\left\{w(a): a \in E_{L}^{*}(S)\right\}$, and $E(S)=\left\{w(a): a \in E^{*}(S)\right\}$, and using the one-to-one property of the restricted circular projections, one has that $E_{R}(S)$ is contained in that part of $B$ to the right of $w_{0}, E_{L}(S)$ is contained in that part of $B$ to the left of $w_{0}$, and $E(S)$ is contained in $B$. Thus from $\S 2.3$ we have an $a_{0} \in F^{*}$ and an $S$ that satisfies all the properties of Theorem 1. This is a contradiction. Thus $F$ must have linear measure zero and the theorem is proved.

2.5. A result that immediately follows from (ii) of Theorem 1 is that for almost every $a \in T^{*}$,

$$
\lim _{\substack{r \rightarrow 0 \\ r \in S}} \frac{L(a, r)}{2 \pi r}=\frac{1}{2} .
$$

From this Corollaries 1 and 2 can easily be established. We are now ready to prove Theorem 2 . We let $\varepsilon$ be an arbitrary positive number. Using the defining properties of the set $T^{*}$ it follows that

$$
\liminf _{r \rightarrow 0} \frac{A(a, r)}{\pi r^{2}} \geq \frac{1}{2}-\varepsilon
$$


for every $a \in T^{*}$. We set

$$
H=\left\{r \in\left(0, r_{a}\right): \frac{L(a, r)}{2 \pi r}>\frac{1}{2}+\varepsilon\right\} .
$$

For almost every $a \in T^{*}, H$ is a measurable set that has density zero at 0 . From $\S 1.3$ we have that

$$
\begin{aligned}
A(a, r) & =\int_{0}^{r} L\left(a, r^{\prime}\right) d r^{\prime} \\
& =\int_{H \cap(0, r)} L\left(a, r^{\prime}\right) d r^{\prime}+\int_{C H \cap(0, r)} L\left(a, r^{\prime}\right) d r^{\prime}, \\
& \text { where } C H \text { denotes the complement of } H, \\
& \leq(2 \pi r) m(H \cap(0, r))+(1 / 2+\varepsilon)\left(\pi r^{2}\right) .
\end{aligned}
$$

Hence

(2) $\quad \limsup _{r \rightarrow 0} \frac{A(a, r)}{\pi r^{2}} \leq 2 \lim _{r \rightarrow 0} \frac{m(H \cap(0, r))}{r}+(1 / 2+\varepsilon)=1 / 2+\varepsilon$.

Using (1) and (2) we have that the limit exists and is $1 / 2$.

\section{REFERENCES}

[1] J. Marafino, Concerning boundary behavior under conformal mappings, Ph.D. Dissertation, University of Wisconsin-Milwaukee, 1984, pp. 33-40.

[2] J. E. McMillan, Boundary behavior of a conformal mapping, Acta Math., 123 (1969), 43-67.

[3] _ On the boundary correspondence under conformal mapping, Duke Math. J., 37 (1970), 725-739.

[4] , Boundary behavior under conformal mapping, Proceedings of the NRL Conference on Classical Function Theory, Mathematics Research Center, Naval Research Laboratory, Washington, D.C. 1970.

[5] M. E. Monroe, Measure and Integration, 2nd ed., Addison-Wesley Publishing Company, (1971), 184, 187, 108-110.

[6] A. Ostrowski, Zur Randverzerrung bei Konformer Abbildung, Prace Mat. Fisycz., 44 (1936), 371-471.

[7] Ch. Pommerenke, Univalent Functions, Vandenhoeck and Ruprecht in Göttingen, 1975.

[8] B. Rodin and S. E. Warschawski, Extremal length and univalent functions, Mathematisch Zetschrift, 153 (1977), 1-17.

[9] M. Tsuji, Potential Theory in Modern Function Theory, Maruzan Company, Tokyo, (1959), 64, 318-322.

Received September 11, 1989. 


\section{PACIFIC JOURNAL OF MATHEMATICS EDITORS}

V. S. VARAdARAJAN

(Managing Editor)

University of California

Los Angeles, CA 90024-1555

Herbert Clemens

University of Utah

Salt Lake City, UT 84112

F. Michael CHRIST

University of California

Los Angeles, CA 90024-1555

ThOMAs ENRIGHT

University of California, San Diego

La Jolla, CA 92093
Nicholas ERCOLANI

University of Arizona

Tucson, AZ 85721

R. FINN

Stanford University

Stanford, CA 94305

VAUGHAN F. R. JoNES

University of California

Berkeley, CA 94720

SteVen KercKhofF

Stanford University

Stanford, CA 94305
C. C. MOORE

University of California

Berkeley, CA 94720

MARTIN SCHARLEMANN

University of California

Santa Barbara, CA 93106

HAROLD STARK

University of California, San Diego

La Jolla, CA 92093

\section{ASSOCIATE EDITORS}
R. Arens
E. F. BeCKenBACH
B. H. NeumanN
F. WolF
(1904-1989)
K. YoshidA (1906-1982)

TIONS

UNIVERSITY OF ARIZONA

UNIVERSITY OF BRITISH COLUMBIA

UNIVERSITY OF OREGON

UNIVERSITY OF SOUTHERN CALIFORNIA

CALIFORNIA INSTITUTE OF TECHNOLOGY

UNIVERSITY OF CALIFORNIA

MONTANA STATE UNIVERSITY

STANFORD UNIVERSITY

UNIVERSITY OF HAWAII

UNIVERSITY OF NEVADA, RENO

UNIVERSITY OF TOKYO

NEW MEXICO STATE UNIVERSITY

UNIVERSITY OF UTAH

OREGON STATE UNIVERSITY

WASHINGTON STATE UNIVERSITY

UNIVERSITY OF WASHINGTON 


\section{Pacific Journal of Mathematics}

Vol. 154, No. $1 \quad$ May, 1992

Richard Arens, Pseudo regular elements in a normed ring $\ldots \ldots \ldots \ldots \ldots 1$

Joan Birman and William W. Menasco, Studying links via closed braids.

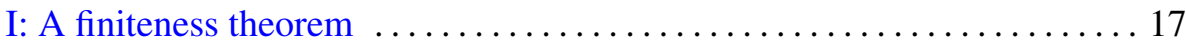

Etsurō Date, Michio Jimbo, Kei Miki and Tetsuji Miwa, Braid group

representations arising from the generalized chiral Potts models ....... 37

Toshihiro Hamachi, A measure theoretical proof of the Connes-Woods

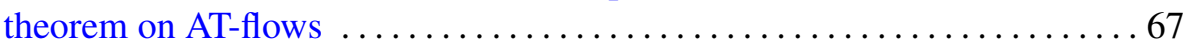

Allen E. Hatcher and Ulrich Oertel, Affine lamination spaces for surfaces ....................................... 87

David Joyner, Simple local trace formulas for unramified $p$-adic groups $\ldots .103$

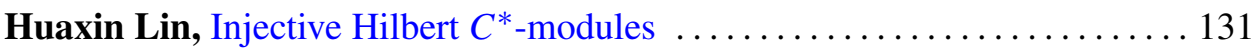

John Marafino, The boundary of a simply connected domain at an inner

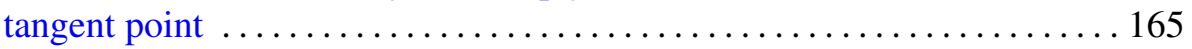

Gonzalo Riera and Rubi Rodriguez, The period matrix of Bring's curve . . 179 